\title{
Composite Higgs theory
}

\section{Florian Goertz*}

Max-Planck-Institut für Kernphysik, Saupfercheckweg 1, 69117 Heidelberg, Germany

E-mail: fgoertz@mpi-hd.mpg.de

I review the idea of realizing the Higgs as a composite pseudo-Nambu-Goldstone boson of a new strongly-interacting sector and collect major constraints on the parameter space of minimal models. Besides limits from electroweak precision tests, LHC searches for resonances and bounds due to Higgs-coupling modifications will be discussed in detail. Finally, the issue of light top partners in these models will be explored, including ways to avoid them which lead to interesting implications for flavor observables.

An Alpine LHC Physics Summit (ALPS2018)

15-20 April, 2018

Obergurgl, Austria

${ }^{*}$ Speaker. 


\section{Introduction}

To understand the stability of the weak scale, $v=\left(\sqrt{2} G_{F}\right)^{-1 / 2} \approx 246 \mathrm{GeV}$, given the presence of large thresholds, such as the Planck scale $M_{\mathrm{Pl}} \sim 10^{19} \mathrm{GeV}$ or the scale of grand unification $M_{\mathrm{GUT}} \sim 10^{15} \mathrm{GeV}$, remains a major task in high energy physics. Beyond this notorious 'hierarchy problem', there are further hierarchies in the flavor sector, in particular in quark masses and mixings and due to the tiny neutrino masses, that can not be explained in the Standard Model (SM) of particle physics. Moreover, in the SM, electroweak symmetry breaking (EWSB) - being responsible for the masses of the known elementary particles - is just parameterized via the Higgs mechanism, but not explained dynamically.

All these issues can be addressed in models where EWSB is not triggered by a fundamental elementary scalar, but ultimately induced by the condensate of a new strong interaction $\langle\hat{O}\rangle$, breaking spontaneously a global symmetry

$$
G \stackrel{\langle\hat{O}\rangle}{\longrightarrow} H
$$

with the electroweak group $G_{\mathrm{EW}} \equiv S U(2)_{L} \times U(1)_{Y}$ embedded in $G$ as a weakly gauged subgroup. The prime example of such a mechanism of dynamical EWSB is realized in Technicolor (TC) theories [1,2], which furnish a good starting point to discuss more recent incarnations of EWSB via similar kinds of dynamics. In the former theories, $G_{\mathrm{EW}}$ is fully broken along with $G$ at the scale $f \sim v$ via condensation, by upscaling the breaking pattern

$$
G=S U(2)_{L} \times S U(2)_{R} \stackrel{\langle\bar{q} q\rangle}{\longrightarrow} H=S U(2)_{V}
$$

of QCD to the EW scale. The latter is now generated from a UV theory via dimensional transmutation, i.e., emerging from a new running coupling becoming strong at a certain scale and triggering the condensation of new (EW charged) 'techni'-fermions, $\left\langle\bar{q}_{\mathrm{TC}} q_{\mathrm{TC}}\right\rangle \neq 0$, which breaks EW symmetry. In this way three Goldstone bosons ('techni-pions'), delivering the longitudinal degrees of freedom for the massive $W$ and $Z$ bosons, emerge by breaking three $S U(2)$ generators (belonging to the coset $G / H)$. The large Goldstone decay constant $f \simeq v=246 \mathrm{GeV} \gg f_{\pi}=92 \mathrm{MeV}$ finally allows for viable weak boson masses. Yet, no physical Higgs boson emerges to explain the 2012 discovery at the LHC [3, 4] (see, however [5, 6, 7, 8]). Moreover, TC theories suffer generically from large corrections to EW precision parameters and it is a challenge to include fermion masses.

All these problems can be solved in composite Higgs $(\mathrm{CH})$ models $[9,10,11]$, which employ a larger coset $G / H$ of broken global symmetries (like $S O(5) / S O(4)$ ) such as to deliver at least four Goldstone bosons that can furnish a full (composite) Higgs doublet. In turn, EW symmetry $G_{\mathrm{EW}} \subset$ $H$ is now broken via the vacuum misalignment mechanism, as detailed in the next section. The Higgs potential emerges radiatively via explicit breaking of the Goldstone symmetry, making the Higgs a pseudo Nambu-Goldstone boson (pNGB). The hierarchy problem is still solved since the Higgs boson is not a fundamental scalar, but is composite above the $\mathrm{TeV}$ scale and its mass is thus saturated in the IR. Moreover, its Goldstone nature provides a reasoning for its lightness compared to other new states. Finally, the presence of a Higgs doublet and the related possibility to separate the EW scale $v$ from the global symmetry breaking scale $f$ allows for a suppression of corrections to (precision) observables by the ratio $\xi \equiv v^{2} / f^{2}$ and eventually for a SM limit, decoupling the 


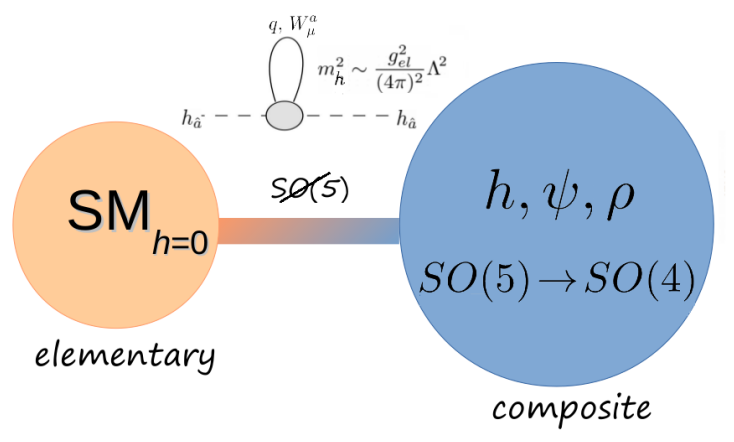

Figure 1: Pictorial representation of the Composite Higgs setup, see text for details.

heavy resonances that reside at $m_{*} \sim g_{*} f$, with $g_{*}<4 \pi$ the coupling of the composite sector (at the price of reintroducing fine-tuning).

The remainder of these notes is organized as follows. In Section 2 we go on to introduce the $\mathrm{CH}$ idea and in particular the vacuum misalignment mechanism and the resulting Higgs potential in more detail. We will also discuss corrections to the Higgs couplings due to its Goldstone nature and briefly introduce the concept of partial compositeness to realize fermion masses. After that, in Section 3, we will explore constraints on $\xi$ following from electroweak precision tests (EWPT), while in Section 4, we will present current limits from LHC searches for heavy resonances and for modified Higgs couplings. $\mathrm{CH}$ models generically predict the presence of anomalously light top partners with masses $m_{t^{\prime}}<f<m_{*}$, which start to be in conflict with null-searches at the LHC. Section 5 contains a discussion of a $\mathrm{CH}$ incarnation that avoids such ultra-light partners, while addressing neutrino masses via a seesaw mechanism, with potentially interesting consequences for flavor physics. Finally, Section 6 contains the conclusions. Although these notes are meant to be self-contained, the emphasis is on providing a condensed overview of the theoretical setup of $\mathrm{CH}$ models and current constraints. For more details on the discussed topics, the reader is referred to the cited literature and comprehensive reviews, such as $[12,13]$.

\section{Vacuum Misalignment and a Composite Higgs}

While several non-minimal cosets have been studied in the literature (see [14] for an overview), here we focus on the minimal composite Higgs realization that features custodial symmetry, i.e., $G / H=S O(5) / S O(4)$ [15]. We thus consider a strongly coupled sector that induces $S O(5) \rightarrow$ $S O(4)\left(\simeq S U(2)_{L} \times S U(2)_{R}\right)$ breaking and contains the composite Higgs as well as bosonic and fermionic resonances, $\rho$ and $\psi$, respectively. This sector is coupled to the SM, which breaks explicitly the global symmetry, since i) the SM gauges only a subgroup of $S O(5)$ and ii) the SM fermions don't fill complete $S O(5)$ representations, and thus induces radiatively a potential for the Goldstone Higgs, which in turn breaks EW symmetry and provides masses for the EW gauge bosons and SM-like fermions. The setup is summarized in pictorial form in Fig. 1.

Explicitly, the minimal description of the composite pNGB Higgs corresponds to a non-linear $\sigma$-model of the $S O(5) / S O(4)$ coset (see, e.g., [16, 17, 18, 19, 20, 21]). The respective Goldstone 

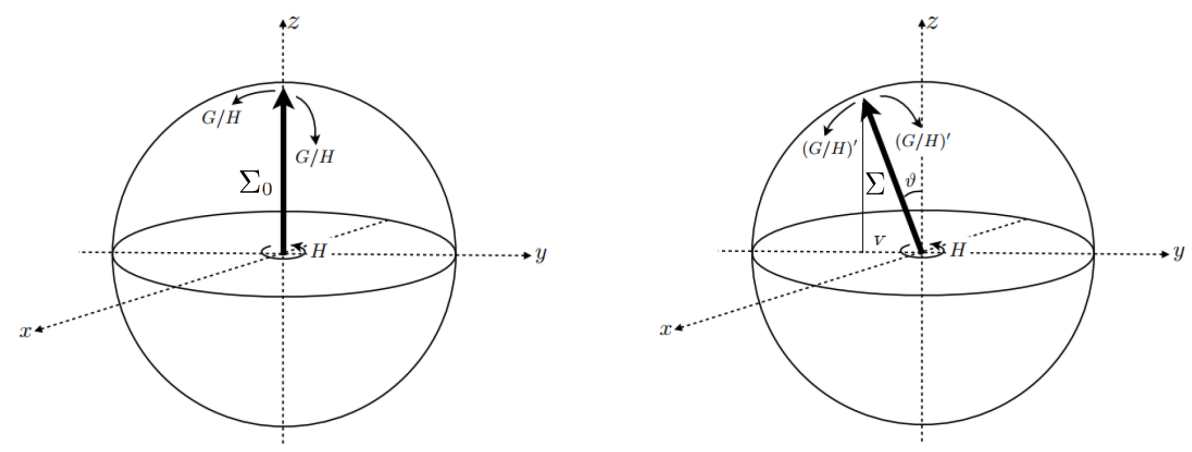

Figure 2: Vacuum misalignment, breaking $G_{\mathrm{EW}} \subset H$. Picture adapted from [24], see text for details.

bosons are parameterized by the $\Sigma$-field

$$
\Sigma=U \Sigma_{0}
$$

which contains the Goldstone matrix

$$
U=\exp \left(i \frac{\sqrt{2}}{f} h_{\hat{a}} T^{\hat{a}}\right),
$$

and corresponds to a local rotation of the ( $S O(4)$-preserving) vacuum configuration

$$
\Sigma_{0}=(0,0,0,0, f)^{T}
$$

The Goldstone fields $h_{\hat{a}}$ are in fact just the 'angular' variables associated to local transformations in the direction of the broken $S O(5)$ generators $T_{i j}^{\hat{a}} \equiv-i / \sqrt{2}\left[\delta_{i}^{\hat{a}} \delta_{j}^{5}-\delta_{j}^{\hat{a}} \delta_{i}^{5}\right]$, and feature the correct quantum numbers to furnish a Higgs doublet. The corresponding Lagrangian, replacing the Higgs sector in the SM, reads

$$
\mathscr{L}_{\Sigma}=\frac{1}{2}\left(D_{\mu} \Sigma\right)^{T} D^{\mu} \Sigma
$$

where $D_{\mu}=\partial_{\mu}-i g^{\prime} Y B_{\mu}-i g T^{i} W_{\mu}^{i}$ is the gauge-covariant derivative, which induces the couplings of the composite Higgs to the SM gauge fields.

Although this minimal description is not complete since it does for example not include the composite resonances $\rho, \Psi$ of the new strongly coupled sector, it allows to derive already several characteristic predictions of the $\mathrm{CH}$ scenario, as we will see below. More UV complete models, including this next threshold (i.e., the heavy resonances) have been constructed in the literature, such as the 5D holographic duals [22, 15] or the (deconstructed) 2-site / 3-site models [18] (see also [23]), and the reader is referred to the corresponding articles for more details. Still, we will at least also introduce a layer of fermionic resonances later when we discuss the realization of the fermion sector via partial compositeness.

Before deriving first predictions of the scenario let us review in more detail how EW is finally broken in $\mathrm{CH}$ models, following the geometrical picture in Fig. 2. The spontaneous breaking of $G$ triggered by strong dynamics leaves - without explicit $G$ breaking - a global symmetry $H$ 
unbroken, with the vacuum $\Sigma_{0}$ pointing in a direction orthogonal to $H$ (see left panel) and the Goldstone bosons of the $G / H$ coset being exactly massless. However, due to explicit $G$-breaking via gauging of $G_{\mathrm{EW}} \subset H \subset G$ and couplings to the SM fermions (see below), the Goldstone-Higgs develops a potential and a vacuum expectation value (vev) $\left\langle h_{\hat{a}}^{2}\right\rangle>0$, breaking $G_{\mathrm{EW}} \subset H$ by shifting the true vacuum $\langle\Sigma\rangle$ with respect to the $H$-preserving $\Sigma_{0}$ by an angle

$$
\vartheta \equiv\langle h\rangle / f,
$$

where $h \equiv \sqrt{\left(h_{\hat{a}}\right)^{2}}$. This is visualized in the right panel of Fig. 2. The amount of breaking of EW symmetry now corresponds to the projection of the shifted vacuum onto the $H$-plane

$$
v=f \sin \vartheta, \quad f=\left|\Sigma_{0}\right|,
$$

with $v \approx 246 \mathrm{GeV}$ the EW vev. The latter measures the misalignment of the true vacuum with respect to $\Sigma_{0}$ and the corresponding mechanism is referred to as vacuum misalignment mechanism $[9,10,11]$. As we sill see below, the challenge now becomes to generate a small value

$$
\xi=\frac{v^{2}}{f^{2}}=\sin ^{2} \vartheta \ll 1,
$$

such as to abandon the TC limit $\xi \rightarrow 1$ and suppress corrections to SM predictions, scaling with $\xi$.

Higgs couplings In fact, we are now ready to have a first look on the couplings of the pNGB Higgs to the SM-like gauge bosons. At low energies we can conveniently describe the properties of the latter (in the background of $\Sigma$ ) using symmetries [15, 12]. Promoting the full global $S O(5) \times$ $U(1)_{X}$ of the strong sector to a gauge symmetry ${ }^{1}$, the most general (quadratic) Lagrangian takes the form

$$
\mathscr{L}_{\text {eff }}^{V}=\frac{1}{2}\left(P_{T}\right)^{\mu v}\left[\Pi_{0}^{X}\left(q^{2}\right) X_{\mu} X_{v}+\Pi_{0}\left(q^{2}\right) \operatorname{Tr}\left(A_{\mu} A_{v}\right)+\Pi_{1}\left(q^{2}\right) \Sigma A_{\mu} A_{v} \Sigma^{T}\right],
$$

with $X$ and $A_{\mu}$ the $U(1)_{X}$ and $S O(5)$ gauge bosons, respectively, $\left(P_{T}\right)^{\mu v} \equiv \eta^{\mu \nu}-q^{\mu} q^{v} / q^{2}$, and $\Sigma$ is treated as a classical background (with vanishing momentum).

Using symmetries and results valid for large number of 'colors' $N$ in the strong sector [25, 26, 27], one can derive explicit results for the form factors $\Pi\left(q^{2}\right)$, that encode the strong dynamics, expanding them for low momenta. In particular, one finds $\Pi_{1}(0)=f^{2}$ and, using the properties of the $S O(5) / S O(4)$ generators $T^{\hat{a}}$ as well as switching of the unphysical gauge fields, we finally obtain, following [12]

$$
\begin{aligned}
\mathscr{L}_{\text {eff }}^{V} & =\frac{f^{2}}{8} \sin ^{2}\left(\frac{\langle h\rangle+h}{f}\right)\left(W_{\mu}^{i} W^{i \mu}-2 W_{\mu}^{3} B^{\mu}+B_{\mu} B^{\mu}\right)+\cdots \\
& =\left(1+2 \sqrt{1-\xi} \frac{h}{v}+(1-2 \xi) \frac{h^{2}}{v^{2}}+\cdots\right)\left(m_{W}^{2} W_{\mu}^{+} W^{-\mu}+\frac{m_{Z}^{2}}{2} Z_{\mu} Z^{\mu}\right)+\cdots,
\end{aligned}
$$

where we have absorbed the gauge couplings into the normalization of the corresponding fields in the intermediate steps. We observe that the couplings of the pNGB Higgs to gauge bosons are

\footnotetext{
${ }^{1}$ The additional $U(1)_{X}$ factor is needed to allow for viable hypercharges of the SM fermions and we will later turn of the spurious gauge degrees of freedom.
} 

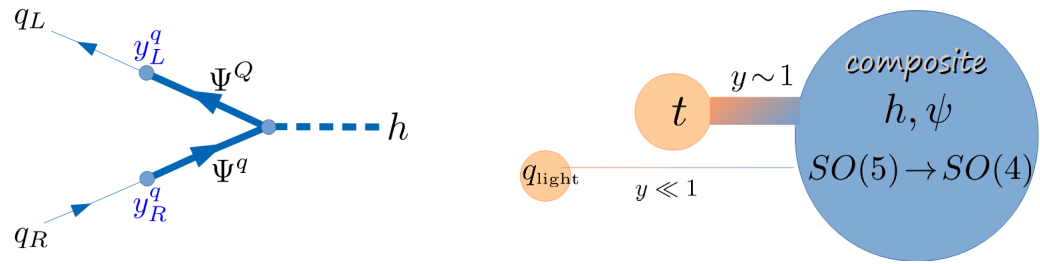

Figure 3: Generation of fermion masses via partial compositeness, see text for details.

generically reduced compared to the corresponding interactions in the $\mathrm{SM}^{2}$ For the couplings of one (two) Higgs bosons to EW gauge fields, $g_{h V V}\left(g_{h h V V}\right)$, we obtain

$$
g_{h V V}=\sqrt{1-\xi} g_{h V V}^{\mathrm{SM}}, \quad g_{h h V V}=(1-2 \xi) g_{h h V V}^{\mathrm{SM}},
$$

which approach the SM values for $\xi \rightarrow 0$.

Partial compositeness and the Higgs potential We now turn to the implementation of fermions in the $\mathrm{CH}$ framework. It turns out that the framework of partial compositeness is particularly suited to realize fermion masses, delivering even an explanation for the large hierarchies that are observed among them $[29,15,22,30]$. Focusing on the quark sector, one basically assumes that the elementary SM-like fields $q_{L, R}$ mix linearly with composite resonances $\Psi_{L, R}^{Q, q}$, with the corresponding mass-mixing Lagrangian reading [21] (see also [18, 30, 31])

$$
\begin{aligned}
\mathscr{L}_{\text {mass }}^{\Psi}= & -y_{L}^{q} f \bar{q}_{L} \Delta_{L}^{q} \Psi_{R}^{Q}-y_{R}^{q} f \bar{q}_{R} \Delta_{R}^{q} \Psi_{L}^{q} \\
& -\sum_{f, f^{\prime}=Q, q} m_{\Psi}^{f f^{\prime}} \bar{\Psi}_{L}^{f} \Psi_{R}^{f^{\prime}}-f \sum_{f, f^{\prime}=Q, q} Y_{f f^{\prime}} \bar{\Psi}_{L}^{f} \frac{\Sigma \Sigma^{T}}{f^{2}} \Psi_{R}^{f^{\prime}}+\text { h.c. }
\end{aligned}
$$

Here, we assumed the composite resonances to reside in the fundamental representation 5 of $S O(5)$ - otherwise the Yukawa couplings in the strong sector $\sim Y_{f f^{\prime}}$ have a different form - and the objects $\Delta_{L, R}^{q}$ project out the components of $\Psi^{Q, q}$ that can couple to the SM fermions in a $G_{\mathrm{EW}}$ invariant way. Note that, in the literature, the minimal (i.e., $S O(5) / S O(4)$ ) composite Higgs model with fermions in the $\mathbf{5}$ is denoted as $\mathrm{MCHM}_{5}$.

From Eq. (2.11) follows that, after diagonalization, the SM-like quarks now correspond to a mixture of elementary and composite fields and the quark masses take the form

$$
m_{q} \sim y_{L}^{q} y_{R}^{q} Y_{Q q} \frac{v f^{2}}{m_{\Psi}^{Q Q} m_{\Psi}^{q q}},
$$

which can also be obtained from integrating out the heavy resonances in the diagram in the left panel of Fig. 3. Now, hierarchically different degrees of compositeness $y_{L, R}^{q}$ of the quarks lead to hierarchical mass eigenvalues (and mixings), see the right panel of the figure, and the former in fact arise in strongly coupled theories via renormalization group running from small differences in

\footnotetext{
${ }^{2}$ See [28] for a discussion on the universality of these results in the IR, i.e., their independence of the concrete coset.
} 
anomalous dimensions of the associated composite operators [15]. In this way, $\mathrm{CH}$ models address - besides the gauge hierarchy problem - also the flavor puzzle. ${ }^{3}$

The $\Delta_{L, R}^{q}$ in Eq. (2.11) explicitly break the $S O(5)$ Goldstone symmetry and thus generate a potential for the pNGB Higgs, which is in general a combination of trigonometric functions of $h / f$. It turns out that the coefficients of these functions need to cancel to (at least) the order $\sin ^{2}(v / f) \ll 1$ to allow for $0<v<<f$, because in general the vacuum, once shifted, tends to be maximally misaligned, $v \sim f$, with respect to the the $S O(4)$ preserving one, due to the explicit symmetry breaking $[15,18,21]$. Clearly, the most important contribution comes from the field with the largest compositeness, which is the heavy top quark, and here and in the following we will neglect the subleading contributions from lighter quarks (and gauge bosons).

In the end, also the Higgs mass will be proportional to the Goldstone symmetry breaking $\left(\sim y_{L, R}^{t}\right)$, and after a careful evaluation one obtains for the $\operatorname{MCHM}_{5}[18,37,21]$

$$
m_{h} \sim y_{t}^{2} v \sim \frac{m_{T}^{0}}{f} m_{t}
$$

where we employed Eq. (2.12) and used the fact that $y_{L}^{t} \sim y_{R}^{t} \equiv y_{t}$ and that $Y_{Q q}$ can be expressed in terms of resonance masses [37], while $m_{T}^{0} \equiv \min \left(m_{\Psi}\right)$ is the mass of the lightest 'top-partner' resonance. Since one expects the latter to reside in general above the scale $f$, we observe that generically the Higgs boson is expected to be heavier than the top quark. A phenomenologically viable $m_{h} \sim 125 \mathrm{GeV}$ requires - at least in the simplest $\mathrm{CH}$ realizations - the lightest top-sector resonance to feature a mass $m_{T}^{0}<f[30,37,38,35,23]$. This is thus in particular much lighter than the vector resonances but allows in turn for a viable top mass with a reduced Goldstone symmetry breaking, as needed for a light Higgs. For $f \sim \mathrm{TeV}$, the above estimate is however in tension with LHC null results in resonance searches and, as we will detail below, it is interesting to search for models which avoid the presence of such problematically light top partners. Before exploring in more detail the phenomenology of the latter, we will first collect lower bounds on $f$ from various observables, such as to understand if $\mathrm{CH}$ models can in general (still) be realized at the $1 \mathrm{TeV}$ scale.

\section{Electroweak Precision Tests}

$\mathrm{CH}$ models can be tested in precise extractions of electroweak (pseudo-)observables, such as the $S, T, U$ parameters, which parameterize NP contributions to electroweak vacuum polarization diagrams [39, 40, 41]. Due to custodial symmetry, the tree-level contribution to $T$ vanishes in the MCHM, while the tree-exchange of spin-1 resonances generates a positive [12, 15]

$$
S=2 \pi \xi \Pi_{1}^{\prime}(0) \approx 4 \pi \frac{v^{2}}{m_{\rho}^{2}},
$$

\footnotetext{
${ }^{3}$ In the dual 5D theory the varying degree of compositeness corresponds to different fermionic wave-function localizations in the extra dimension, originating from $\mathscr{O}(1)$ input parameters (see, e.g., [32] and references therein), and the structure closely resembles that of the Froggatt-Nielsen mechanism [33, 34, 35, 36].
} 

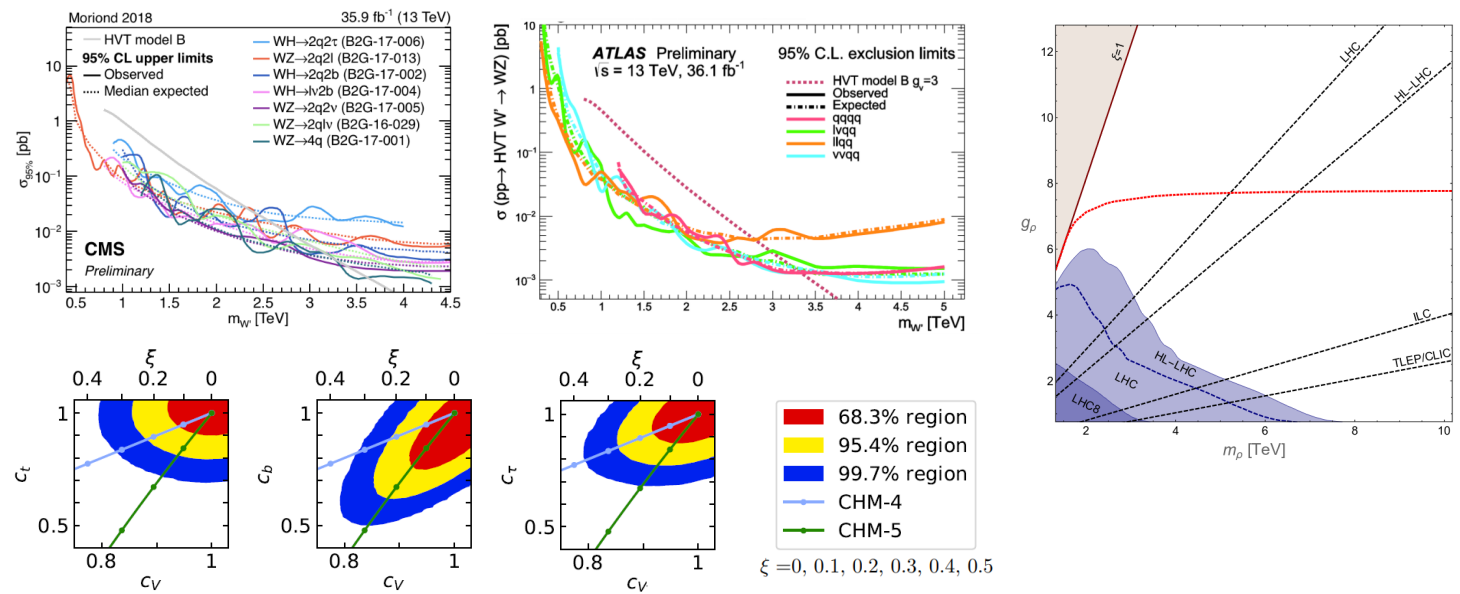

Figure 4: Upper row: Limits from CMS (left) and ATLAS (center) searches for vector resonances [44] and future projections [42] (right). Lower row: Limits on $\xi$ from a fit to Higgs data [49], considering the 2Dplanes of couplings to gauge bosons and top quarks, bottom quarks, and tau leptons, where $c_{X}=g_{h X X} / g_{h X X}^{\mathrm{SM}}$.

which depends on the form factor introduced in Eq. (2.8). Moreover, $m_{\rho} \sim m_{*}$ is the scale of the vector-resonances, ${ }^{4}$ and, as opposed to the TC case, $S$ can become arbitrarily small considering $\xi \ll 1$.

A second important contribution to the electroweak precision parameters arises at one-loop from the fact that the Higgs to gauge-boson couplings are modified [20], $g_{V V h} / g_{V V h}^{\mathrm{SM}}=\sqrt{1-\xi} \neq 1$. One finds [20, 12]

$$
\begin{aligned}
\Delta S & =+\frac{1}{12 \pi} \xi \log \frac{\Lambda^{2}}{m_{h}^{2}}, \\
\Delta T & =-\frac{3}{16 \pi \cos ^{2} \theta_{W}} \xi \log \frac{\Lambda^{2}}{m_{h}^{2}},
\end{aligned}
$$

with $\Lambda \approx 4 \pi f$. Recent global analyses of constraints on the MCHM from EWPT arrive at [42, 43]

$$
\xi \lesssim 0.1 \Leftrightarrow f \gtrsim 800 \mathrm{GeV} @ 95 \% \mathrm{CL} .
$$

\section{LHC Searches}

\subsection{Resonance Searches}

An obvious way to test $\mathrm{CH}$ scenarios is to search for the composite resonances predicted in the setup. We first focus on vector-boson resonances of $Z^{\prime}$ and $W^{\prime}$ type, while fermionic resonances will be discussed further below in Sec. 5. The most promising decay channels of these resonances in $\mathrm{CH}$ models clearly involve the heaviest SM states, i.e., $W, Z, h$ bosons or the top quark.

From the latest CMS and ATLAS searches, presented at Moriond '18 [44], see left plots in Fig. 4, we extract the bound

$$
m_{\rho} \gtrsim(2.5-3.5) \mathrm{TeV} \stackrel{g_{\rho}=3}{\Longleftrightarrow} f \gtrsim(850-1200) \mathrm{GeV},
$$

\footnotetext{
${ }^{4}$ This does not necessarily coincide with the scale of fermionic resonances, since the sectors might in principle be governed by different couplings, $g_{\rho} \neq g_{\Psi}$.
} 
which is based on the assumption $g_{\rho}=3$ and, by now, furnishes a very competitive constraint. In fact, the limits depend sensitively on the couplings of the resonances to the SM fields and have always to be considered with care. A thorough analysis, discussing such effects, has been presented in [42] (see also [45, 46]), where also projections for future colliders are obtained. In the rightmost plot in Fig. 4, the 95\% CL limits from LHC8 as well as projections for the LHC with $300 \mathrm{fb}^{-1}$ and the high-luminosity LHC with $3000 \mathrm{fb}^{-1}$ are presented in the $m_{\rho}-g_{\rho}$ plane, employing dark and light violet, respectively.

\subsection{Higgs Physics}

Another powerful test of the $\mathrm{CH}$ paradigm is to search for deviations in the Higgs couplings, potentially unraveling its Goldstone nature. The couplings to gauge bosons in the MCHM have been given in Eq. (2.10) while those to fermions are more model dependent. For the $\mathrm{MCHM}_{5}$, taking only into account the global shift due to the non-linear nature of the Higgs, we obtain (see, e.g., [47])

$$
g_{h f f}=\frac{1-2 \xi}{\sqrt{1-\xi}} g_{h f f}^{\mathrm{SM}} .
$$

Several groups have performed fits to Higgs data, see, e.g., [48, 49, 50, 51, 52, 53, 54]. In the lower panel of Fig. 4 we display the results of Ref. [49], which provide limits on the $\mathrm{MCHM}_{4}$ and $\mathrm{MCHM}_{5}$ that can be obtained from the intersection of the colored fit regions with the $\mathrm{CH}$ predictions, the latter given by blue and green lines with dots for various values of $\xi$. The generally good agreement with the SM expectations leads to the bound

$$
\xi \lesssim 0.12 \Leftrightarrow f \gtrsim 700 \mathrm{GeV} @ 95 \% \mathrm{CL}
$$

for the $\mathrm{MCHM}_{5}[49,48]$, which starts to become competitive with the one from EWPT. ${ }^{5}$

Note that a detailed comparison of indirect and direct reaches for $\mathrm{CH}$ models has been performed in [42], where the direct limits in the right plot of Fig. 4 have been confronted with projected indirect limits from Higgs-coupling modifications at various colliders, given as dashed lines in the same plot. The latter bound directly the ratio $\xi$ and thus show a linear behavior in the $m_{\rho}-g_{\rho}$ plane (complementary to direct searches), where projected LHC limits reside in the ballpark of $\xi \lesssim 0.1$, while ILC/TLEP/CLIC are expected to reach $\xi \lesssim 10^{-2}-10^{-3}$.

\section{Light Top Partners and Lepton-Flavor Observables}

Before concluding, we finally come back to the issue of light top partners in $\mathrm{CH}$ models. As explained in Sec. 2, we expect $m_{T}^{0} / f \sim m_{h} / m_{t}<1$ in the $\mathrm{MCHM}_{5}$. The corresponding numerical prediction from [21] is given in the left plot in Fig. 5, for $f=800 \mathrm{GeV}$, where the color code depicts the degree of (Barbieri-Giudice) tuning [58]. It turns out that in the $\mathrm{MCHM}_{5}$ in fact a viable Higgs mass (given by the yellow band) requires generically

$$
m_{T}^{0} \lesssim 800 \mathrm{GeV} \text {. }
$$

\footnotetext{
${ }^{5}$ For current limits in other $\mathrm{CH}$ scenarios, see, e.g., [48, 53], as well as [14, 31, 55, 56, 57] which include discussions on effects from fermion mixing.
} 

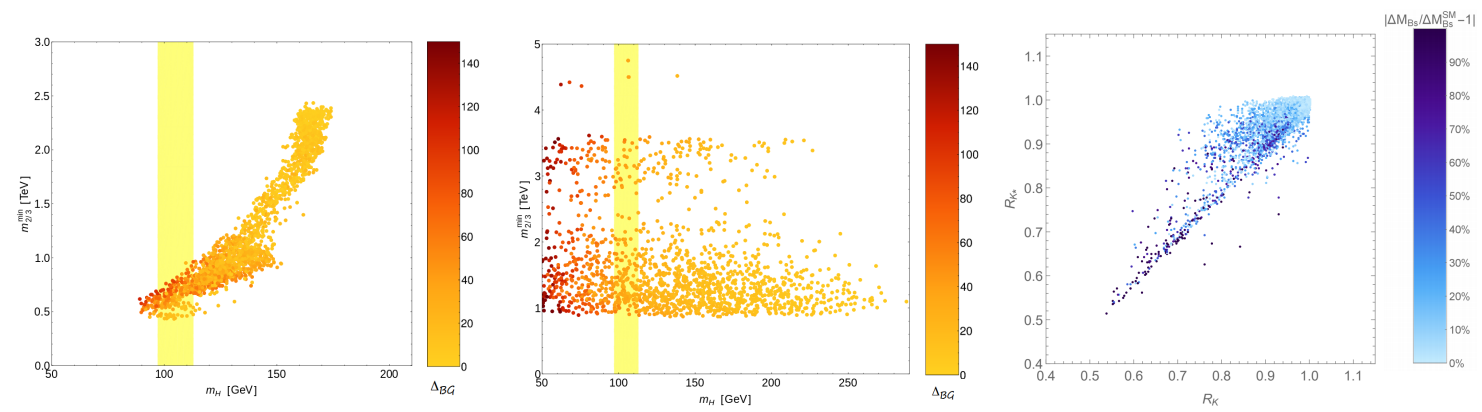

Figure 5: Masses of lightest top partners in the $\mathrm{MCHM}_{5}$ (left) and in the minimal lepton model of [62] (center), where $f=800 \mathrm{GeV}$ as well as predictions in the $R_{K}-R_{K^{*}}$ plane for the latter model.

On the other hand, searches for top partners at ATLAS and CMS are already excluding masses of up to $\gtrsim 1 \mathrm{TeV}[59,60]$, which is starting to become an issue for TeV-scale $\mathrm{CH}$ models.

While the masses of top partners could be raised by using less minimal quark representations, featuring a large number of new states and requiring a rather unmotivated ('ad-hoc') tuning in the Higgs mass [61], an interesting alternative is to consider a minimal implementation of a nontrivial lepton sector in the MCHM. As was shown in [21, 62], in the framework of the type-III seesaw mechanism it is possible to unify also the right-handed charged and neutral leptons in a single representation of the global symmetry, which leads to a highly predictive lepton sector (featuring 2 instead of $4 S O(5)$ multiplets) with in total less degrees of freedom than in standard $\mathrm{CH}$ incarnations. At the same time, the setup predicts a moderate compositeness of the right-handed SM-like charged leptons (driven by the unification with the seesaw fields) [21], which enters in a parametrically enhanced way into the Higgs potential. This non-negligible lepton contribution to the potential allows to raise the masses of the top partners, such as to meet current experimental limits, while a light Higgs remains natural. The numerical results are presented in the central plot of Fig. 5, which visualizes that now the lightest top partners can easily be in the $2-3 \mathrm{TeV}$ range.

Another interesting consequence of the scenario of lepton compositeness is that it predicts a violation of lepton flavor universality (LFU), which can be tested for example at LHCb. In fact, the latter experiment saw hints for a deviation from the SM prediction for the LFU-probing observables $R_{K}$ and $R_{K^{*}}$, which reads $R_{K}^{\mathrm{SM}}=R_{K^{*}}^{\mathrm{SM}}=1$ to good approximation. It turns out that the $\mathrm{CH}$ model of [62] strictly predicts both $R_{K}<1$ and $R_{K^{*}}<1$, see the right plot in Fig. 5 [63], just going in the correct direction to address the experimental tensions, while meeting other constraints from flavor physics $[62,63]$.

\section{Conclusions}

We reviewed the Composite Higgs solution to the gauge hierarchy problem and the flavor puzzle. We collected current constraints on minimal models, covering EWPT, resonance searches and tests of Higgs properties, where the latter two are becoming competitive due to the successful LHC operation. Moreover, we discussed the issue of light top partners in composite models and presented ways to avoid them with interesting consequences for flavor physics. 
Acknowledgments I am grateful to the organizers of ALPS2018 for the invitation and the excellent organization and atmosphere at the workshop.

\section{References}

[1] S. Weinberg, Phys. Rev. D 13 (1976) 974 Addendum: [Phys. Rev. D 19 (1979) 1277].

[2] L. Susskind, Phys. Rev. D 20 (1979) 2619.

[3] S. Chatrchyan et al. [CMS Collaboration], Phys. Lett. B 716 (2012) 30 [arXiv:1207.7235 [hep-ex]].

[4] G. Aad et al. [ATLAS Collaboration], Phys. Lett. B 716 (2012) 1 [arXiv:1207.7214 [hep-ex]].

[5] B. Holdom, Phys. Rev. D 24 (1981) 1441.

[6] W. A. Bardeen, C. N. Leung and S. T. Love, Phys. Rev. Lett. 56 (1986) 1230.

[7] K. Yamawaki, M. Bando and K. i. Matumoto, Phys. Rev. Lett. 56 (1986) 1335.

[8] B. Bellazzini, C. Csaki, J. Hubisz, J. Serra and J. Terning, Eur. Phys. J. C 73 (2013) no.2, 2333 [arXiv:1209.3299 [hep-ph]].

[9] D. B. Kaplan and H. Georgi, Phys. Lett. 136B (1984) 183.

[10] D. B. Kaplan, H. Georgi and S. Dimopoulos, Phys. Lett. 136B (1984) 187.

[11] M. J. Dugan, H. Georgi and D. B. Kaplan, Nucl. Phys. B 254 (1985) 299.

[12] R. Contino, arXiv:1005.4269 [hep-ph].

[13] G. Panico and A. Wulzer, Lect. Notes Phys. 913 (2016) pp.1 [arXiv:1506.01961 [hep-ph]].

[14] B. Bellazzini, C. Csáki and J. Serra, Eur. Phys. J. C 74 (2014) no.5, 2766 [arXiv:1401.2457 [hep-ph]].

[15] K. Agashe, R. Contino and A. Pomarol, Nucl. Phys. B 719 (2005) 165 [hep-ph/0412089].

[16] S. R. Coleman, J. Wess and B. Zumino, Phys. Rev. 177 (1969) 2239.

[17] C. G. Callan, Jr., S. R. Coleman, J. Wess and B. Zumino, Phys. Rev. 177 (1969) 2247.

[18] G. Panico and A. Wulzer, JHEP 1109 (2011) 135 [arXiv:1106.2719 [hep-ph]].

[19] G. F. Giudice, C. Grojean, A. Pomarol and R. Rattazzi, JHEP 0706 (2007) 045 [hep-ph/0703164].

[20] R. Barbieri, B. Bellazzini, V. S. Rychkov and A. Varagnolo, Phys. Rev. D 76 (2007) 115008 [arXiv:0706.0432 [hep-ph]].

[21] A. Carmona and F. Goertz, JHEP 1505 (2015) 002 [arXiv:1410.8555 [hep-ph]].

[22] R. Contino, Y. Nomura and A. Pomarol, Nucl. Phys. B 671 (2003) 148 [hep-ph/0306259].

[23] S. De Curtis, M. Redi and A. Tesi, JHEP 1204 (2012) 042 [arXiv:1110.1613 [hep-ph]].

[24] A. Azatov and J. Galloway, Int. J. Mod. Phys. A 28 (2013) 1330004 [arXiv:1212.1380 [hep-ph]].

[25] G. ’t Hooft, Nucl. Phys. B 72 (1974) 461.

[26] G. 't Hooft, Nucl. Phys. B 75 (1974) 461.

[27] E. Witten, Nucl. Phys. B 160 (1979) 57.

[28] D. Liu, I. Low and Z. Yin, arXiv:1805.00489 [hep-ph].

[29] D. B. Kaplan, Nucl. Phys. B 365 (1991) 259. 
[30] R. Contino, L. Da Rold and A. Pomarol, Phys. Rev. D 75 (2007) 055014 [hep-ph/0612048].

[31] A. Azatov and J. Galloway, Phys. Rev. D 85 (2012) 055013 [arXiv:1110.5646 [hep-ph]].

[32] T. Gherghetta, arXiv:1008.2570 [hep-ph].

[33] Y. Grossman and M. Neubert, Phys. Lett. B 474 (2000) 361 [hep-ph/9912408].

[34] S. J. Huber and Q. Shafi, Phys. Lett. B 498 (2001) 256 [hep-ph/0010195].

[35] C. Csaki, A. Falkowski and A. Weiler, JHEP 0809 (2008) 008 [arXiv:0804.1954 [hep-ph]].

[36] S. Casagrande, F. Goertz, U. Haisch, M. Neubert and T. Pfoh, JHEP 0810 (2008) 094 [arXiv:0807.4937 [hep-ph]].

[37] O. Matsedonskyi, G. Panico and A. Wulzer, JHEP 1301 (2013) 164 [arXiv:1204.6333 [hep-ph]].

[38] A. Pomarol and F. Riva, JHEP 1208 (2012) 135 [arXiv:1205.6434 [hep-ph]].

[39] M. E. Peskin and T. Takeuchi, Phys. Rev. Lett. 65 (1990) 964.

[40] M. E. Peskin and T. Takeuchi, Phys. Rev. D 46 (1992) 381.

[41] J. Haller, A. Hoecker, R. Kogler, K. Mönig, T. Peiffer and J. Stelzer, Eur. Phys. J. C 78 (2018) no.8, 675 [arXiv: 1803.01853 [hep-ph]].

[42] A. Thamm, R. Torre and A. Wulzer, JHEP 1507 (2015) 100 [arXiv:1502.01701 [hep-ph]].

[43] D. Ghosh, M. Salvarezza and F. Senia, Nucl. Phys. B 914 (2017) 346 [arXiv:1511.08235 [hep-ph]].

[44] ATLAS and CMS talks on searches for heavy resonances at Moriond EW 2018.

[45] M. Low, A. Tesi and L. T. Wang, Phys. Rev. D 92 (2015) no.8, 085019 [arXiv:1507.07557 [hep-ph]].

[46] C. Niehoff, P. Stangl and D. M. Straub, JHEP 1601 (2016) 119 [arXiv:1508.00569 [hep-ph]].

[47] R. Contino, M. Ghezzi, C. Grojean, M. Muhlleitner and M. Spira, JHEP 1307 (2013) 035 [arXiv:1303.3876 [hep-ph]].

[48] V. Sanz and J. Setford, Adv. High Energy Phys. 2018 (2018) 7168480 [arXiv:1703.10190 [hep-ph]].

[49] J. de Blas, O. Eberhardt and C. Krause, JHEP 1807 (2018) 048 [arXiv:1803.00939 [hep-ph]].

[50] A. Falkowski, Pramana 87 (2016) no.3, 39 [arXiv:1505.00046 [hep-ph]].

[51] T. Corbett, O. J. P. Eboli, D. Goncalves, J. Gonzalez-Fraile, T. Plehn and M. Rauch, JHEP 1508 (2015) 156 [arXiv:1505.05516 [hep-ph]].

[52] S. Fichet and G. Moreau, Nucl. Phys. B 905 (2016) 391 [arXiv:1509.00472 [hep-ph]].

[53] A. Banerjee, G. Bhattacharyya, N. Kumar and T. S. Ray, JHEP 1803 (2018) 062 [arXiv:1712.07494 [hep-ph]].

[54] J. Ellis, C. W. Murphy, V. Sanz and T. You, JHEP 1806 (2018) 146 [arXiv:1803.03252 [hep-ph]].

[55] A. Carmona and F. Goertz, JHEP 1304 (2013) 163 [arXiv:1301.5856 [hep-ph]].

[56] D. Barducci, A. Belyaev, M. S. Brown, S. De Curtis, S. Moretti and G. M. Pruna, JHEP 1309 (2013) 047 [arXiv:1302.2371 [hep-ph]].

[57] M. Carena, L. Da Rold and E. Pontón, JHEP 1406 (2014) 159 [arXiv:1402.2987 [hep-ph]].

[58] R. Barbieri and G. F. Giudice, Nucl. Phys. B 306 (1988) 63.

[59] M. Aaboud et al. [ATLAS Collaboration], JHEP 1807 (2018) 089 [arXiv:1803.09678 [hep-ex]]. 
[60] A. M. Sirunyan et al. [CMS Collaboration], JHEP 1711 (2017) 085 [arXiv:1706.03408 [hep-ex]].

[61] G. Panico, M. Redi, A. Tesi and A. Wulzer, JHEP 1303 (2013) 051 [arXiv:1210.7114 [hep-ph]].

[62] A. Carmona and F. Goertz, Phys. Rev. Lett. 116 (2016) no.25, 251801 [arXiv:1510.07658 [hep-ph]].

[63] A. Carmona and F. Goertz, arXiv:1712.02536 [hep-ph]. 